\title{
Identification of a novel biomarker for pyridoxine-dependent epilepsy: Implications for newborn screening
}

Michael F. Wempe ${ }^{1,{ }^{*}}$, Amit Kumar ${ }^{1}$, Vijay Kumar ${ }^{1}$, Yu J. Choi ${ }^{1}$, Michael A. Swanson², Marisa W. Friederich $^{2}$, Keith Hyland ${ }^{3}$, Wyatt W. Yue ${ }^{4}$, Johan L.K. Van Hove ${ }^{2, a}$, Curtis R. Coughlin II ${ }^{2, a,{ }^{* *}}$

${ }^{1}$ School of Pharmacy, Department of Pharmaceutical Sciences, University of Colorado, Anschutz Medical Campus, Aurora, CO, USA

${ }^{2}$ Department of Pediatrics, Section of Clinical Genetics and Metabolism, University of Colorado School of Medicine, Aurora, CO USA

${ }^{3}$ Medical Neurogenetics Laboratories, LLC, Atlanta, GA, USA

${ }^{4}$ Structural Genomics Consortium, Nuffield Department of Clinical Medicine, University of Oxford, Oxford, UK

${ }^{a}$ These authors contributed equally to this work

Correspondence:

* Michael.wempe@ucdenver.edu (MF Wempe)

${ }^{* *}$ Curtis.coughlin@ucdenver.edu (CR Coughlin II)

Word count Abstract: 242

Word count Text: 4395

Number of Figures: 3

Number of Tables: 0 


\begin{abstract}
:
Pyridoxine-dependent epilepsy (PDE) is often characterized as an early onset epileptic encephalopathy with dramatic clinical improvement following pyridoxine supplementation. Unfortunately, not all patients present with classic neonatal seizures or respond to an initial pyridoxine trial, which can result in the under diagnosis of this treatable disorder. Restriction of lysine intake and transport is associated with improved neurologic outcomes, although treatment should be started in the first year of life to be effective. Because of the documented diagnostic delay and benefit of early treatment, we aimed to develop a newborn screening method for PDE. Previous studies have demonstrated the accumulation of $\Delta^{1}$-piperideine-6carboxylate and $\alpha$-aminoadipic semialdehyde in individuals with PDE, although these metabolites are unstable at room temperature limiting their utility for newborn screening. As a result, we sought to identify a biomarker that could be applied to current newborn screening paradigms. We identified a novel metabolite, 6-oxo-pipecolate, which accumulates in substantial amounts in blood, plasma, urine and cerebral spinal fluid of individuals with PDE. Using a stable isotope labeled internal standard, we developed a non-derivatized liquid chromatography tandem mass spectrometry-based method to quantify 6-oxo-pipecolate. This method replicates the analytical techniques used in many laboratories and could be used with few modifications in newborn screening programs. Furthermore, 6-oxo-pipecolate was measurable in urine for four months even when stored at room temperature. Herein, we report a novel biomarker for PDE that is stable at room temperature and can be quantified using current newborn screening techniques.
\end{abstract}

Synopsis: This study identified a novel biomarker in patients with PDE, which can be applied to current newborn screening paradigms 
Details of the contribution of individual authors: MFW, JVH and CRC conceived of study design, reviewed data and drafted the manuscript. MFW, AK, VK, YC, MAF and MAS produced, reviewed and interpreted laboratory data. $\mathrm{CRC}$ and $\mathrm{KH}$ provided patient samples. WWY and MAS produced specialized purified human enzymes. All authors critically reviewed and contributed to the final manuscript and agreed to be accountable for the final version of the manuscript.

Name of corresponding authors: Michael F. Wempe and Curtis R. Coughlin II

A competing interest statement: A Kumar, V Kumar, Y Choi, M Swanson, M Friederich, W Yue declare that they have no conflict of interest. $\mathrm{K}$ Hyland receives salary and has owner interest in Medical Neurogenetics - a company that performs clinical testing for PDE. M Wempe, J Van Hove and C Coughlin have a patent application for diagnostic markers for PDE pending.

Details of funding: This work was supported by NIH/NCATS Colorado CTSA Grant Number UL1 TR001082 and the US-Based patient organization: Pyridoxine Dependent Epilepsy Foundation.

Details of ethics approval: Ethics approval was received from the Colorado Multiple Institutional Review Board (COMIRB \#16-0585; \#16-1184).

A patient consent statement: Individual written informed consent was obtained from patients

\section{Documentation of approval from the Institutional Committee for Care and Use of} Laboratory Animals: Not applicable

Keywords: ALDH7A1; Alpha aminoadipic semialdehyde; Pyridoxine dependent epilepsy; 6oxo-pipecolate; 6-hydroxypiperidine 2-carboxylic acid 
Introduction: Pyridoxine-dependent epilepsy (PDE) is often characterized as a treatment refractory epileptic encephalopathy with dramatic clinical or electroencephalogram improvement after pyridoxine supplementation (Hunt et al. 1954). Many patients achieve adequate seizure control with pyridoxine alone. However, $75 \%$ of individuals treated with pyridoxine monotherapy have significant intellectual disability (ID) and developmental delay (Basura et al. 2009; Bok et al. 2012). The degree of ID does not correlate with age of diagnosis or degree of seizure control, suggesting that the cognitive impairment is not solely a result of epilepsy.

PDE is caused by a deficiency of $\alpha$-aminoadipic semialdehyde ( $\alpha$-AASA) dehydrogenase, and results in the accumulation of $\alpha-A A S A$ and $\Delta^{1}$-piperideine-6-carboxylate ( $\left.\Delta^{1}-\mathrm{P} 6 \mathrm{C}\right)$, which are considered to be in equilibrium (Mills et al. 2006; Struys and Jakobs 2010; Struys et al. 2012). There are two lysine oxidation pathways that converge at $\alpha$-AASA. In the saccharopine pathway, the single polypeptide bi-functional enzyme a-aminoadipic-semialdehyde synthase (AASS) couples lysine with 2-oxoglutarate to produce saccharopine in the lysine ketoglutarate reductase domain followed by the saccharopine dehydrogenase domain which converts saccharopine to glutamate and $\alpha-A A S A$ (Vashishtha et al. 2015). In the pipecolate pathway, Llysine is converted into $\Delta^{1}$-piperideine-2-carboxylate $\left(\Delta^{1}-\mathrm{P} 2 \mathrm{C}\right)$ via a yet to be identified enzyme and mechanism, although $\alpha$-transamination has been proposed (Hallen et al. 2013). The brain enzyme $\Delta^{1}-\mathrm{P} 2 \mathrm{C}$ reductase $(\mathrm{P} 2 \mathrm{CR})$ then reduces $\Delta^{1}-\mathrm{P} 2 \mathrm{C}$ to L-pipecolic acid (PIP) (Rothstein and Miller 1954; Garweg et al. 1980; Hallen et al. 2013), which is then oxidized via L-pipecolate oxidase resulting in $\Delta^{1}$-P6C (IJlst et al. 2000). Of note, $\Delta^{1}$-P6C can be converted backwards into PIP via pyrroline-5-carboxylate reductase (Meng et al. 2006; Struys et al. 2014). Both the pipecolate pathway and the saccharopine pathway converge at $\Delta^{1}$-P6C and $\alpha-A A S A$, and $\alpha$ AASA is further oxidized to 2-aminoadipic acid (AAA) by a-AASA dehydrogenase (Mills et al. 2006; Chan et al. 2011).

Recent treatment paradigms have attempted to reduce the accumulation of $\alpha$-AASA and $\Delta^{1}$ P6C through a lysine-restricted diet and competitive inhibition of lysine-transport (van Karnebeek et al. 2012; Mercimek-Mahmutoglu et al. 2014; Coughlin et al. 2015; Mahajnah et al. 2016; Yuzyuk et al. 2016b). These lysine restriction therapies result in significant reduction of $\alpha$ $\mathrm{AASA} / \triangle^{1}-\mathrm{P} 6 \mathrm{C}$ and improved cognitive outcome, although treatment must be initiated within the first year of life for optimal developmental outcome (Al Teneiji et al. 2017). Unfortunately the diagnosis of PDE is often delayed (Basura et al. 2009; Falsaperla et al. 2018). The delay in 
diagnosis limits the effectiveness of lysine reduction therapies and is a major contributor to the overall morbidity of the disease.

Recent estimates suggest a disease incidence of approximately 1:60,000 live births (Coughlin et al. 2018). This is similar to other diseases that currently undergo newborn screening such as galactosemia (1:50,000 live births) and biotinidase deficiency (1:60,000 live births) (Suzuki et al. 2001; Porta et al. 2017). Because of the benefit of early treatment, documented long diagnostic delay, and sufficiently high frequency of disease incidence, we sought to develop a newborn screening method for PDE. Previous attempts at newborn screening have documented the unstable nature of $\alpha-A A S A$ and $\Delta^{1}$-P6C at room temperature (Struys et al. 2012; Jung et al. 2013; Mathew et al. 2018). As a result, we attempted to stabilize $\alpha-A A S A$ by derivatization with 2,4-dinitrophenylhydrazine (2,4-DNP), but no reaction product was observed. The subsequent characterization of various metabolites allowed for the identification of a novel metabolite, 6oxo-pipecolate (6-oxopiperidine 2 carboxylic acid, 6-oxo-PIP), which accumulates in blood, plasma, urine and cerebral spinal fluid (CSF) of patients with PDE. This new biomarker is stable at room temperature and can be quantified using current newborn screening techniques, which would make it amiable for newborn screening.

\section{Methods}

Human Subjects: Three subjects were recruited via the Inherited Metabolic Disease Clinic at Children's Hospital of Colorado on an IRB-approved study (COMIRB \#16-0585). Inclusion criteria included PDE due to $\alpha$-AASA dehydrogenase deficiency documented by bi-allelic mutations in $A L D H 7 A 1$ or elevation of $\alpha-A A S A / \triangle^{1}-\mathrm{P} 6 \mathrm{C}$. Blood, plasma, and urine samples were obtained from three subjects. A CSF sample was obtained from a fourth subject. A control group consisted of anonymous, discarded plasma, urine and CSF originally obtained for clinical testing at the chemistry laboratory at the Children's Hospital of Colorado (COMIRB \#16-1184).

Chemicals and reagents: Deuterium oxide $\left(\mathrm{D}_{2} \mathrm{O}\right)$, deuterated sulfuric acid $\left(\mathrm{D}_{2} \mathrm{SO}_{4}\right)$, deuterated chloride (DCl), deuterated acetic acid $\left(\mathrm{CH}_{3} \mathrm{CO}_{2} \mathrm{D}\right)$, deuterated ethanol $\left(\mathrm{CH}_{3} \mathrm{CH}_{2}-\mathrm{OD}\right)$, deuterated ammonium hydroxide ( $\left.\mathrm{ND}_{4} \mathrm{OD}\right), \mathrm{L}$-allysine ethylene acetal (AEA), L-lysine, L-glutamine, AAA, PIP, L-amino acid oxidase from Crotalus adamanteus (Type IV), Lysine Oxidase from Trichoderma viride, Catalase from bovine liver, L-saccharopine, NAD $+2,4-D N P$, uridine 5'diphosphoglucuronic acid trisodium salt, amberlyst-15 and 6-oxo-PIP were purchased from Sigma-Aldrich Chemical Company (St. Louis, MO). D,L-2-Amino-1,6-hexanedioic-2,5,5-d3 (D3- 
AAA) was acquired from CDN Isotopes (Quebec, Canada), and all other reagents were procured from Fisher Scientific (Pittsburgh, PA). Control human plasma, blood and urine were purchased from Bioreclamation LLC (Westbury, NY). Human liver cytosol, S9 and microsomal subcellular fractions were procured from Xenotech LLC (Kansas City, KS).

Synthesis of standard material: Standards were synthesized through the deprotection of Lallysine ethylene acetal (AEA). In the Amberlyst method, AEA (11.4 mg) was dissolved in $\mathrm{H}_{2} \mathrm{O}$ $(1.0 \mathrm{ml})$ with $76 \mathrm{mg}$ Amberlyst-15 and stirred for $30 \mathrm{~min}$ at RT. The liquid was transferred to a new glass vial, the beads were then washed with $\mathrm{NH}_{4} \mathrm{OH}(2 \times 1.0 \mathrm{~mL})$, and the contents combined as previously described (Sadilkova et al. 2009). The material was then concentrated under a $\mathrm{N}_{2}$ stream and reconstituted with water to a final concentration of $30 \mathrm{mM}$. In the acid catalysed method, AEA was similarly dissolved in water and $6 \mathrm{~N} \mathrm{HCl}$ or $\mathrm{H}_{2} \mathrm{SO}_{4}$ was added in amounts described in Table S2, stirred for $30 \mathrm{~min}$, and $\mathrm{pH}$-adjusted with $\mathrm{NaHCO}_{3}$ and diluted with water to a concentration of $20 \mathrm{mM}$ product.

For deuterated experiments in the Amberlyst method, AEA (71 mg) was dissolved in $\mathrm{D}_{2} \mathrm{O}(2.0$ $\mathrm{mL}$ ) and Amberlyst-15 (721 mg) added and stirred at RT (6 hr) at which point the $\mathrm{D}_{2} \mathrm{O}$ was removed and $\mathrm{ND}_{4} \mathrm{OD}(1.0 \mathrm{~mL})$ added, stirred (15 min) and solution removed, and nuclear magnetic resonance (NMR) spectra collected. For deuterated experiments in the acid catalyzed method, AEA was dissolved in $\mathrm{D}_{2} \mathrm{O}$ and added either $\mathrm{DCl}$ or $\mathrm{D}_{2} \mathrm{SO}_{4}$ to initiate the reaction, and we then collected the ${ }^{1} \mathrm{H}-\mathrm{NMR}$ and ${ }^{13} \mathrm{C}-\mathrm{NMR}$ spectra.

Aldehyde detection via 2,4-dinitrophenyl hydrazine: The 2,4-DNP reagent procedure was prepared as previously described with minor modifications (Ruekberg and Rossoni 2005). 500 $\mathrm{mg}$ of 2,4-DNP was weighed out into a $25 \mathrm{ml}$ Erlenmeyer flask and $4.0 \mathrm{ml}$ water and $11.0 \mathrm{ml}$ ethanol were added and stirred while $2.5 \mathrm{ml} \mathrm{H}_{2} \mathrm{SO}_{4}$ was added dropwise. The exothermic reaction was cooled with an ice bath. The contents were then allowed to warm to room temperature and stirred until completely into solution. $200 \mu \mathrm{L}$ of this solution was added to the analyte solution either during or after the generation from AEA.

\section{Hydrogen-1 and Carbon-13 nuclear magnetic resonance spectroscopy experiments: AEA} (10 mg) was dissolved in $700 \mu \mathrm{L} \mathrm{D}_{2} \mathrm{O}$ followed by addition of either $50 \mu \mathrm{LCl}$ or $\mathrm{D}_{2} \mathrm{SO}_{4}$, and immediately transferred to a NMR tube and ${ }^{1} \mathrm{H}$ and ${ }^{13} \mathrm{C}$ spectra collected using a $400 \mathrm{MHz}$ Bruker NMR, Avance III 400; the ${ }^{1} \mathrm{H}$-NMR spectra were collected at $400 \mathrm{MHz}$ while the ${ }^{13} \mathrm{C}-\mathrm{NMR}$ 
spectra were collected at $100 \mathrm{MHz}$ and the chemical shifts are reported in ppm. Additional NMR experiments were prepared via $\mathrm{D}_{2} \mathrm{O}$ via $\mathrm{DCl}$ conditions followed by $\mathrm{pH}$ adjustment through the addition of $\mathrm{NaHCO}_{3}$ (1.5 equivalents) to produce $\Delta^{1}-\mathrm{P} 6 \mathrm{C}$.

Liquid chromatography tandem mass spectrometry experiments: Lysine metabolites were first analyzed based on previously published liquid chromatography tandem mass spectrometry (LC-MS/MS) methods (Struys and Jakobs 2007; Yuzyuk et al. 2016a). As the goal of this study was to replicate current newborn screening testing modalities, we focused on a non-derivatized MS/MS analytical method (Nagy et al. 2003). Samples were analyzed via electrospray ionization in positive ion mode (ESI +) using an Applied Biosystems Sciex 4000 (Applied Biosystems; Foster City, CA) equipped with a Shimadzu HPLC (Shimadzu Scientific Instruments, Inc.; Columbia, MD) and a Leap auto-sampler (LEAP Technologies; Carrboro, NC). Methanol (MeOH): acetonitrile (ACN) $200 \mu \mathrm{L}$ 1:1 mixture was added to $100 \mu \mathrm{L}$ of plasma, blood, urine, CSF or standard materials. Plasma and blood samples were centrifuged at $10,000 \mathrm{rpm}$ and the supernatants used. Urine and CSF samples required no additional processing. The liquid chromatography separation of $10 \mu \mathrm{L}$ sample was done on two tandem Zorbax-C8 $150 \times 4.6 \mathrm{~mm}$ 5 micron columns with a Zorbax-C8 guard column (Agilent Technologies) operated at $40^{\circ} \mathrm{C}$ with a $0.4 \mathrm{~mL} / \mathrm{min}$ flow-rate, using the mobile phases $\mathrm{A}\left(10 \mathrm{mM} \mathrm{NH}_{4} \mathrm{OAc}, 0.1 \%\right.$ formic acid in $\mathrm{H}_{2} \mathrm{O}$ ) for $12.0 \mathrm{mM}$, linear ramp to $95 \%$ mobile phase $B(1: 1 \mathrm{ACN}: \mathrm{MeOH})$ at $16.0 \mathrm{~min}$, held for 11.5 min, followed by ramping back to $A$ and held for a total run time of $32.0 \mathrm{~min}$. Samples were analyzed using the following conditions: i) ion-spray voltage of $5500 \mathrm{~V}$; ii) temperature, $450{ }^{\circ} \mathrm{C}$; iii) collision using nitrogen gas with curtain gas (CUR) set at 10 and collisionally activated dissociation (CAD) set at 12; iv) ion source gas one (GS1) and two (GS2) were set at 30; v) entrance potential was set at $10 \mathrm{~V}$; vi) quadruple one (Q1) and (Q3) were set on unit resolution; vii) dwell time was set at $200 \mathrm{msec}$. The retention times, mass transitions and other settings are listed in Table S1.

Synthesis of standards: The internal standard d3-3,5,5-6-oxopipecolate (D3-oxo-PIP) was synthesized by refluxing $\mathrm{D}_{3}-\mathrm{AAA}$ (40 $\mathrm{mg}, 0.24 \mathrm{mmol}$ ) in $20 \%$ mono-deuteroacetic acid $\left(\mathrm{CH}_{3} \mathrm{COOD}\right) / \mathrm{D}_{2} \mathrm{O}(1.0 \mathrm{~mL})$ at $108^{\circ} \mathrm{C}$ for $3.5 \mathrm{hr}$, followed by solvent evaporation under reduced pressure and drying on high vacuum for $1.0 \mathrm{hr}$, after which deuterated ethanol $\left(\mathrm{CH}_{3} \mathrm{CH}_{2} \mathrm{OD} ; 10\right.$ $\mathrm{mL}$ ) was added, stirred, and filtered twice. The combined two filtrate solutions were concentrated on reduced pressure and dried on high vacuum (4 hr) resulting in $14 \mathrm{mg}$ deuterated 6-oxo-PIP of $>97 \%$ purity by NMR and LC-MS/MS analysis. 
Enzymatic production of lysine metabolism products: To evaluate the products of lysine metabolism through the pipecolate pathway we synthesized the intermediate $\Delta^{1}-\mathrm{P} 2 \mathrm{C}$ enzymatically as follows. Lysine $50 \mathrm{mM}$ with $100 \mu \mathrm{L}$ bovine catalase (12.1 mg catalase powder in $1.0 \mathrm{~mL}$ PBS buffer $\mathrm{pH} 7.4$ ) and $50 \mu \mathrm{L}$ T. viridae lysine oxidase (acquired enzyme diluted with $250 \mu \mathrm{L}$ water) in $750 \mu \mathrm{L}$ PBS were incubated at $37^{\circ} \mathrm{C}$ for $1 \mathrm{hr}$, and $100 \mu \mathrm{L}$ samples were obtained at 0.5, 2, 5, 10, 15, 30, 45 and $60 \mathrm{~min}$, added to $300 \mu \mathrm{L}$ 1:1 MeOH:ACN, and analyzed via LC-MS/MS. To identify the reaction product of the saccharopine pathways, recombinant saccharopine dehydrogenase domain of human AASS-SDH was expressed in insect Sf9 cells and purified by chromatography methods (Yue W.W et al., manuscript in preparation). Stock saccharopine (10 mM; $100 \mu \mathrm{L} ; 1.0 \mathrm{mM}$ final) and NAD ${ }^{+}(100 \mu \mathrm{L} ; 1.0 \mathrm{mM}$ final) were added to PBS (790 $\mu \mathrm{L} ; \mathrm{pH} 7.4)$, and after preincubation at $37^{\circ} \mathrm{C}$ for $5 \mathrm{~min}$, the reaction was initiated by addition of $10 \mu \mathrm{L}$ purified human AASS-SDH and $100 \mu \mathrm{L}$ samples taken at $0.5 \mathrm{~min}, 5,10$ and 15 min, were diluted with $400 \mu \mathrm{L}$ 1:1 MeOH:ACN and analyzed by LC-MS/MS.

a-AASA dehydrogenase was expressed in BL21 StarTM (DE3) E. coli (ThermoFisher) cultures transformed with the pET15b vector containing ALDH7A1 (Coulter-Mackie et al. 2012), and harvested $3 \mathrm{hr}$ after induction by $1.0 \mathrm{mM}$ IPTG. Expression culture lysate was combined with 2.2 $\mathrm{mM} \mathrm{NAD}^{+} 0.6 \mathrm{mM}$ and $\Delta^{1}$-P6C for $5 \mathrm{~min}$ at $30^{\circ} \mathrm{C}$ (Coulter-Mackie et al. 2012, 2014; Coughlin et al. 2018) and quenched by adding $100 \mu \mathrm{L}$ glacial acetic acid to reaction solution (500 $\mu \mathrm{L})$.

Cytosolic and mitochondrial metabolism of lysine metabolites: Human liver cytosol $100 \mu \mathrm{L}$ $(10 \mathrm{mg} / \mathrm{mL})$ or S9 fraction $100 \mu \mathrm{L}(20 \mathrm{mg} / \mathrm{mL})$ and $100 \mu \mathrm{L} \mathrm{NAD}{ }^{+}(1.2 \mathrm{mM}$ final $)$ in $400 \mu \mathrm{L}$ PBS $\mathrm{pH} 7.4$ were preincubated at $37^{\circ} \mathrm{C}$ for $5 \mathrm{~min}$, followed by addition of $100 \mu \mathrm{L} \Delta^{1}$-P6C $(100 \mu \mathrm{M})$; $100 \mu \mathrm{L}$ samples were taken at 0.5, 5, 20 and $50 \mathrm{~min}$, added to $300 \mu \mathrm{L}$ 1:1 MeOH:ACN and analysed by LC-MS/MS. To probe potential phase II metabolism, $100 \mu \mathrm{L}$ human liver microsomes (20 mg protein $/ \mathrm{mL}$ ) were similarly incubated with 4.0 mM UDPGA.

Human liver mitochondria were freshly prepared by homogenization of a human liver sample with a Teflon pestle in 5\% weight to volume Zheng buffer (Zheng et al. 1990), and after centrifugation at $5600 \mathrm{~g}$ for $5 \mathrm{~min}$, the supernatant was transferred to a centrifuge tube; this procedure was repeated on the resuspended pellet a second time. Both supernatants were combined, centrifuged at 37,500 $\mathrm{g}$ for $5 \mathrm{~min}$, the resulting pellet resuspended in Zheng buffer, homogenized and similarly pelleted again, and resuspended in $300 \mu \mathrm{L}$ Zheng buffer to provide a 
mitochondrial preparation, with protein concentration determined using the Bio-Rad Protein Assay. To access the mitochondrial matrix, the homogenate was subjected to 3 freeze-thaw cycles, followed by $2 \times 7$ bursts of sonication on ice using a Branson 450 digital sonifier with a 2-inch cup horn at 30\% amplitude. Of this mitochondrial preparation $100 \mu \mathrm{L}$ was similarly incubated with $\Delta^{1}-\mathrm{P} 6 \mathrm{C}$ and analyzed as above.

Biomarker stability studies: In a 4-month stability study, fresh urine samples (400 $\mu \mathrm{L})$ from two subjects were aliquoted into $1.5 \mathrm{~mL}$ Eppendorf tubes and stored either at room temperature $\left(22 \pm 2^{\circ} \mathrm{C}\right)$, freezer $\left(-13 \pm 3^{\circ} \mathrm{C}\right)$ or deep freezer $\left(-77 \pm 3^{\circ} \mathrm{C}\right)$ and sampled over four-months.

Computational calculations: Chemical structures were drawn using ChemDraw Ultra 6.0.1 and z-matrix utilized to perform calculations via Gaussian G98w. Structures were optimized using molecular mechanics and calculations performed at the HF/6-31G level of theory, followed by B3LYP//HF/6-311++G.

\section{Results}

Identity of the standards made from L-allysine ethylene acetal: Following preparation from AEA by the Amberlyst $15 / \mathrm{NH}_{4} \mathrm{OH}$ method, two peaks were noted on LC-MS/MS with $\mathrm{t}_{\mathrm{R}}=8.5$ min and mass transitions $128.2 \rightarrow 82.0$, and $55.0 \mathrm{~m} / \mathrm{z}$ presumed to be $\Delta^{1}-\mathrm{P} 6 \mathrm{C}$ (Fig. S1A), and with $\mathrm{t}_{\mathrm{R}}=9.0 \mathrm{~min}$ and mass transitions $146.3 \rightarrow 128.0,82.1$ and $55.1 \mathrm{~m} / \mathrm{z}$ presumed to be $\alpha$ AASA (Fig. S1B). The relative intensities of these two peaks varied with the catalyst used (Amberlyst-15 versus $\mathrm{H}_{2} \mathrm{SO}_{4}$ or $\mathrm{HCl}$ ), time, and the $\mathrm{pH}$ of the solution (Table S2). Acid catalyzed deprotection without $\mathrm{pH}$ neutralization resulted in a single signal at $t_{R} 9.0$ min enabling further characterization through DNPH reactivity and NMR spectroscopy.

Aldehyde detection via 2,4-dinitrophenyl hydrazine: 2,4-DNP reagent was added to positive controls including 4-ethoxybenzaldehyde (3.4 mg; red precipitant), octanal (4.2 mg; yellow precipitant), hexanal (3.8 mg; yellow precipitant) and acetone (5.6 mg; yellow precipitant). Compounds were prepared in $1.5 \mathrm{~mL}$ Eppendorf tubes as 50\% ethanol solutions (400 $\mu \mathrm{L})$ and 2,4-DNP reagent $(200 \mu \mathrm{L})$ was added and mixed. Two different AEA 2,4-DNP reactions were performed with AEA. First, AEA (5.0 mg) was diluted with water $(150 \mu \mathrm{L})$ and 50\% ethanol (150 $\mu \mathrm{L}$ ). $200 \mu \mathrm{L}$ of the 2,4-DNP reagent (containing sulfuric acid) was immediately added and a chemical reaction was noted with formation of a yellow precipitant, which was characterized by LC-MS/MS to have a mass consistent with 2,4-DNP-AASA adduct (Fig. S2A). Next, we allowed 
the AEA reaction to occur for $1.0 \mathrm{hr}$ followed by $50 \%$ ethanol $(150 \mu \mathrm{L})$. Once this reaction was completed, $200 \mu \mathrm{L}$ of the 2,4-DNP reagent was added without evidence of a chemical reaction (Fig. S2B). The reaction was warmed at $37^{\circ} \mathrm{C}$ and allowed to cool without evidence of solid formation (chemical reaction). This data suggested that the aldehyde, which is present during formation, rapidly cyclized and is no longer present in the final product. To verify this assumption, NMR spectroscopy was performed.

\section{Hydrogen-1 and Carbon-13 nuclear magnetic resonance spectroscopy experiments: ${ }^{1} \mathrm{H}$} and ${ }^{13} \mathrm{C}$-NMR studies were performed to confirm the identity of the starting material AEA using acid catalyzed deprotection without neutralization, which resulted in a single peak at $t_{R} 9.0 \mathrm{~min}$. This was initially presumed to be $\alpha$-AASA but did not react with 2,4-DNP. In the acid catalyzed reaction product of AEA we did not observe an aldehyde $-\mathrm{CH}$ signal around 9-11 ppm in the ${ }^{1} \mathrm{H}$ NMR, but rather we observed a downfield signal at 8.2 ppm (Fig. S3A). We interpreted this signal as the $-\mathrm{CH}$ alkene within $\Delta^{1}$ - $\mathrm{P} 6 \mathrm{C}$ similar to the alkene $-\mathrm{CH}$ signal at $7.7 \mathrm{ppm}$ published for DL- $\Delta^{1}$-pyrroline-5-carboxylic acid (Farrant et al. 2001). In addition, the ${ }^{13} \mathrm{C}$-NMR spectrum shows signals at 80.5 and 80.7 ppm being consistent with the secondary alcohol carbons consistent with 6-hydroxy-pipecolate (piperidine-6-hydroxy-2-caroxylate, 6-OH-PIP) with the 146 parent mass (Fig. S3B). Thus, the ${ }^{1} \mathrm{H}-\mathrm{NMR}$ data, ${ }^{13} \mathrm{C}-\mathrm{NMR}$ and LC-MS/MS data suggest a mixture of compounds, which co-elutes at $t_{R} 9.0$ min with an apparent 1:2 ratio via LC-MS/MS with respective $\mathrm{m} / \mathrm{z}$ of 128 and 146 (Fig. S4). Despite a number of experimental conditions including the use of a C18, silica, amine and cyano columns for the LC-MS/MS experiments, we could not separate what we believe is a $\Delta^{1}-\mathrm{P} 6 \mathrm{C} / 6-\mathrm{OH}-\mathrm{PIP}$ mixture.

As noted above, $\alpha$-AASA and $\Delta^{1}-\mathrm{P} 6 \mathrm{C}$ are in equilibrium although this equilibrium is not well understood (Struys et al. 2012). Previous studies have noted the difficulty in detecting $\alpha$-AASA, which is supported by our data presented here, and suggested the equilibrium favors $\Delta^{1}-\mathrm{P} 6 \mathrm{C}$ (Pena et al. 2016). Computational calculations show that the cyclic forms of 6-OH-PIP Cis and 6-OH-PIP Trans are energetically more stable than the linear aldehyde a-AASA by 9.1 and 5.9 $\mathrm{kcal} / \mathrm{mol}$ (Table S3). Intramolecular cyclization of $\alpha-A A S A$ without loss of water to $\Delta^{1}-\mathrm{P} 6 \mathrm{C} / 6-\mathrm{OH}-$ PIP is an energetically favorable process.

We assigned the identity of the peak at $t_{R} 8.5$ min as most consistent with $\Delta^{2}$-piperideine-6carboxylate $\left(\Delta^{2}-\mathrm{P} 6 \mathrm{C}\right)$, based on the mass transition and NMR spectrum, and deuterated experiments, although absence of an authentic standard prevented absolute identification. 
There was evidence of the $\Delta^{2}$-P6C double bond in the ${ }^{13} \mathrm{C}-\mathrm{NMR}$ spectra (108.6 and $\left.127.9 \mathrm{ppm}\right)$ (Fig. S5). These signals where were not present when one uses $\mathrm{DCl}$ or $\mathrm{D}_{2} \mathrm{SO}_{4}$ conditions. This data also suggests that $\Delta^{2}-\mathrm{P} 6 \mathrm{C}$ is in equilibrium with $\Delta^{1}$-P6C, with $\Delta^{2}$-P6C forming more under neutral to basic conditions. $\Delta^{2}-\mathrm{P} 6 \mathrm{C}$ can convert back to $\Delta^{1}-\mathrm{P} 6 \mathrm{C}$ but with incorporation of deuterium under $\mathrm{D}_{2} \mathrm{O}$. Thus, the two peaks of observed reaction products correspond to $\Delta^{2}-\mathrm{P} 6 \mathrm{C}$ at $t_{R} 8.6 \mathrm{~min}$, and a mixture of $\Delta^{1}-\mathrm{P} 6 \mathrm{C}$ and $6-\mathrm{OH}-\mathrm{PIP}$ at $9.0 \mathrm{~min}$ (Fig. S6), with the relative amounts a function of the conditions, time and $\mathrm{pH}$ adjustment used to deprotect the acetal.

\section{Reaction products and substrates for enzyme activities in the lysine metabolic pathways:} Incubation of saccharopine with purified AASS-SDH and NAD resulted in products that on LCMS/MS were also consistent with $\Delta^{1}$-P6C/6-OH-PIP. Incubation of purified $\alpha$-AASA dehydrogenase with the $\Delta^{1}$-P6C/6-OH-PIP mixture without $\alpha$-AASA readily produced AAA, indicating that this enzyme catalyzes the ring opening and subsequent oxidation of $\Delta^{1}-\mathrm{P} 6 \mathrm{C} / 6$ OH-PIP. Given that 6-OH-PIP has a secondary alcohol, an alternative pathway to AAA could exist by first oxidation of the alcohol of 6-OH-PIP to 6-oxo-PIP, an amide, followed by hydrolytic ring opening to afford AAA. Incubating the $\Delta^{1}-\mathrm{P} 6 \mathrm{C} / 6-\mathrm{OH}-\mathrm{PIP}$ mixture with a fresh purified mitochondrial preparation only afforded AAA, but incubation of $\Delta^{1}-\mathrm{P} 6 \mathrm{C} / 6-\mathrm{OH}-\mathrm{PIP}$ with human liver cytosol and with the $\mathrm{S9}$ fraction in the presence of $\mathrm{NAD}^{+}$showed formation of $\mathrm{AAA}$ and small amounts of 6-oxo-PIP (Fig. S7). Albeit small, the formation of 6-oxo-PIP raises the possibility of a second pathway. To evaluate if the next step the hydrolytic ring opening of 6-oxoPIP to afford AAA could proceed, we incubated 6-oxo-PIP with human cytosol, human S9 and human plasma (e.g. esterase activity) incubations, but none of these experiments showed a conversion of 6-oxo-PIP to AAA.

To generate authentic standards for the pipecolic pathway, incubation of lysine with L-amino acid oxidase and catalase resulted in small amounts of $\Delta^{1}-\mathrm{P} 2 \mathrm{C}$ and, presumptively, the hydrated product piperidine-2-hydroxy-2-carboxylate (P2H2C) (Fig. S8). Incubation with lysine oxidase and catalase was far more efficient in producing $\Delta^{1}-\mathrm{P} 2 \mathrm{C}$ and $\mathrm{P} 2 \mathrm{H} 2 \mathrm{C}$.

Quantitative LC-MS/MS method of the lysine pathway metabolites: To evaluate the identified metabolites of the lysine pathway, we expanded the analytic LC-MS/MS method to incorporate lysine, glutamine, AAA, PIP, $\Delta^{1}-\mathrm{P} 2 \mathrm{C} / \mathrm{P} 2 \mathrm{H} 2 \mathrm{C}, \Delta^{2}-\mathrm{P} 6 \mathrm{C}, \Delta^{1}-\mathrm{P} 6 \mathrm{C} / 6-\mathrm{OH}-\mathrm{PIP}$, and 6-oxoPIP (Fig. S9). There are two peaks for 6-oxo-PIP representing the enol and keto form. For quantification, deuterated d3-AAA and d3-6-oxo-PIP were used as internal standards. The limit 
of detection (LOD) for $\Delta^{1}$-P6C/6-OH-PIP was $1.0 \mu \mathrm{M}$ and the limit of quantitation (LOQ) was 2.0 $\mu \mathrm{M}$ (Fig. S10A). The LOD and LOQ for 6-OXO-PIP were $2.0 \mathrm{mM}$ and $4.0 \mathrm{mM}$, respectively (Fig. $\mathrm{S} 10 \mathrm{~B})$. A standard curve between 0.5 and $500 \mu \mathrm{M}$ for quantification of $\Delta^{1}$-P6C/6-OH-PIP with internal standard d3-AAA had a correlation coefficient $>0.99$, and for quantification of 6-0xo-PIP with the internal standard d3-6-oxo-PIP had a correlation coefficient $>0.985$.

Human subjects: In all blood, plasma, and urine samples of subjects affected with PDE we detected AAA, PIP, $\Delta^{1}$-P6C/6-OH-PIP, and 6-oxo-PIP (Fig. S11), whereas in samples form 14 control subjects, we only detected AAA and PIP. In two affected subjects, the amount of 6-oxo$\mathrm{PIP}$ in urine was $156.8 \mu \mathrm{mol} / \mathrm{mg}$ creatinine and $122.2 \mu \mathrm{mol} / \mathrm{mg}$ creatinine and for $\Delta^{1}-\mathrm{P} 6 \mathrm{C} / 6-\mathrm{OH}$ $\mathrm{PIP}$ was $8.5 \mu \mathrm{mol} / \mathrm{mg}$ creatinine and $7.5 \mu \mathrm{mol} / \mathrm{mg}$ creatinine. In plasma, the concentration of 6 oxo-PIP was $2.7 \pm 0.1 \mu \mathrm{M}$ and $4.1 \pm 0.1 \mu \mathrm{M}$ and the concentration of $\Delta^{1}-\mathrm{P} 6 \mathrm{C} / 6-\mathrm{OH}-\mathrm{PIP}$ was $1.1 \pm 0.1 \mu \mathrm{M}$ and $3.0 \pm 0.1 \mu \mathrm{M}$ for patients 1 and 2 respectively. In CSF from a subject affected with PDE we also identified $\Delta^{2}$-P6C and a small but clear peak of $\Delta^{1}$-P2C/P2H2C (Fig. S12) in addition to $\Delta^{1}$-P6C/6-OH-PIP and 6-oxo-PIP. In three control CSF samples, AAA and PIP were observed but $\Delta^{1}$-P6C/6-OH-PIP, 6-oxo-PIP, $\Delta^{2}-\mathrm{P} 6 \mathrm{C}$ and $\Delta^{1}-\mathrm{P} 2 \mathrm{C} / \mathrm{P} 2 \mathrm{H} 2 \mathrm{C}$ were absent.

Biomarker stability studies: As a primary aim of this research was to evaluate biomarkers suitable for newborn screening, we analyzed blood spots (Whatman 903® Lot \# W-041) from two subjects and controls. In blood spots stored at room temperature, the signal of $\Delta^{1}-\mathrm{P} 6 \mathrm{C} / 6$ $\mathrm{OH}-\mathrm{PIP}$ rapidly degraded over time, and became undetectable after a few days. In contrast, 6 oxo-PIP was noted in the initial blood spot (Fig. 1). A freeze-thaw study and a 4-month stability study was done in urine samples from subjects 1 and 2 (Table S4). The original concentrations of $\Delta^{1}$-P6C/6-OH-PIP for subject 1 and 2 were $16.1 \pm 1.9 \mu \mathrm{M}$ and $27.1 \pm 2.0 \mu \mathrm{M}$, respectively. At room temperature, $\Delta^{1}$-P6C/6-OH-PIP degraded within a couple of days with only trace amounts remaining after two weeks. When in the freezer or the deep freezer, $\Delta^{1}-\mathrm{P} 6 \mathrm{C} / 6-\mathrm{OH}-\mathrm{PIP}$ decayed moderately. After 126 days, there was a 53-64\% loss of $\Delta^{1}-\mathrm{P} 6 \mathrm{C} / 6-\mathrm{OH}-\mathrm{PIP}$ at $-13 \pm 3^{\circ} \mathrm{C}$ and $47-$ $48 \%$ loss at $-77 \pm 3^{\circ} \mathrm{C}$. The initial 6-oxo-PIP concentrations observed in subject 1 and 2 were $298 \pm 23 \mu \mathrm{M}$ and $440 \pm 4 \mu \mathrm{M}$, respectively. 6-oxo-PIP showed an initial 20-21\% decrease within the first two weeks regardless of storage temperature with, at room temperate, a slower rate of degradation displaying only a 43\% and 33\% decrease at 126 days for subject 1 and 2 , respectively. Thus, at room temperature, 6-oxo-PIP was considerably more stable than $\Delta^{1}$ P6C/6-OH-PIP (Fig. 2). 
Discussion: Herein we describe a novel biomarker (6-oxo-PIP) for PDE, which has significant implications for newborn screening of this treatable disease. Previous attempts at newborn screening were limited as the primary biomarkers $\Delta^{1}$-P6C and $\alpha-A A S A$ degraded rapidly at room temperature (Jung et al. 2013; Mathew et al. 2018). In our study, 6-oxo-PIP was measurable for up to four months in urine samples stored at room temperature. Stability at room temperature is essential as the current newborn screen paradigm relies on sampled to be collected, dried, and shipped at room temperature. Using a stable isotope labeled internal standard, we developed a non-derivatized method LC-MS/MS based method to quantify 6-oxoPIP in subjects and controls. We suggest that screening for PDE could be added to the current newborn screen paradigm where samples are collected on filter paper cards, dried and shipped at room temperature, and analyzed via non-derivatized MS/MS analytical methods. Further studies are needed to establish the sensitivity and specificity of 6-oxo-PIP for PDE. To our knowledge, 6-oxo-PIP has not been reported in patients with PDE or other defects of lysine metabolism, although it has been identified in Penicillium chrysogenum (Brundidge et al. 1980; Henriksen et al. 1998).

The presence of 6-oxo-PIP was suggested by the presence of 6-OH-PIP as an intermediate step between $\Delta^{1}$-P6C and $\alpha$-AASA (Fig. 3). 6-OH-PIP represents the cyclization of $\alpha$-AASA without loss of water and the simple addition of water across the $\mathrm{C}=\mathrm{N}$ bond in $\Delta^{1}-\mathrm{P} 6 \mathrm{C}$. Oxidation of the secondary alcohol in 6-OH-PIP results in the formation 6-oxo-PIP. Our combined NMR, mass spectrometry and DNP reactivity data suggests that a mixture of $\Delta^{1}$ $\mathrm{P} 6 \mathrm{C} / 6-\mathrm{OH}-\mathrm{PIP}$ exists, although this mixture could not be separated despite the use of multiple analytical methods. We suggest that these two metabolites are in a very rapid equilibrium in any aqueous condition. The initial NMR experiments are consistent with both a double bond and a hydroxyl group. This suggests that both interacting products are present in the original standard and not solely occurring with the analytical mass spectrometry method.

Various mutations in ALDH7A1 result in the accumulation of $\Delta^{1}-\mathrm{P} 6 \mathrm{C} / 6-\mathrm{OH}-\mathrm{PIP}$ and $\alpha-\mathrm{AASA}$. $\alpha$ AASA dehydrogenase is present in both mitochondria and cytosol. When $\Delta^{1}-\mathrm{P} 6 \mathrm{C} / 6-\mathrm{OH}-\mathrm{PIP}$ accumulates, it appears to be a substrate of a dehydrogenase using NAD+ located in the cytosol, but not in the mitochondria, and resulting in the formation of 6-oxo-PIP. The exact identity of this enzyme still needs to be determined. An alternative possibility includes the formation of pipecolate from the incubated $\Delta^{1}$-P6C and a direct oxidation of pipecolate to 6-oxo- 
PIP. Although this appears less likely, it is not excluded by the data presented in this paper. The 6-oxo-PIP formed cannot be further metabolized to AAA and accumulates in patients with PDE.

Of note, we identified a small but distinct peak of $\Delta^{1}-\mathrm{P} 2 \mathrm{C} / \mathrm{P} 2 \mathrm{H} 2 \mathrm{C}$ in the CSF of a single affected subject, which was not identified in blood, plasma or urine of affected patients. If confirmed, the presence of $\Delta^{1}-\mathrm{P} 2 \mathrm{C} / \mathrm{P} 2 \mathrm{H} 2 \mathrm{C}$ may indicate a role for the pipecolate pathway in human brain lysine metabolism. The respective contributions of each pathway cannot be determined from this study, and has been subject of controversy (Sauer et al. 2011; Posset et al. 2015; Pena et al. 2017). Further studies are needed to determine which of the many accumulating metabolites contributes to the neurological symptoms of PDE.

Conclusion: In summary, we report 6-OH-PIP as an intermediate metabolite between $\Delta^{1}$-P6C and $\alpha$-AASA in lysine oxidation. A minor cytosolic enzymatic pathway allows oxidation to 6-oxoPIP. Accumulation of 6-0xo-PIP was identified in the blood, plasma, urine and CSF of subjects with PDE and represents a novel biomarker. We present an analytical method for quantification of this new biomarker using stable isotope dilution of LC-MS/MS. Unlike previously identified biomarkers for PDE, 6-oxo-PIP was relatively stable at room temperature. Stability of a biomarker at room temperature is essential to add screening for PDE into existing newborn screening paradigms. The clinical utility of 6-oxo-PIP will need to be defined in a future study of multiple subjects with PDE. 
Acknowledgements This work was supported by NIH/NCATS Colorado CTSA Grant Number UL1 TR001082 and the US-Based patient organization: Pyridoxine Dependent Epilepsy Foundation. 


\section{Figure Legends}

\section{Figure 1: Identification of biomarkers in a dried filter paper spot}

Legend: $\quad$ A dried filter paper (Whatman 903® Lot \# W-041) was impregnated with $200 \mu \mathrm{L}$ of blood from a patient with pyridoxin-dependent epilepsy. Using a non-derivatized liquid chromatography tandem mass spectrometry method, we identified pipecolic acid, the equilibrium of $\Delta^{1}$-piperideine-6-carboxylate and 6-hydroxy-pipecolate ( $\left.\Delta^{1}-\mathrm{P} 6 \mathrm{C} / 6-\mathrm{OH}-\mathrm{PIP}\right)$ and 6 oxo-pipecolate (6-oxo-PIP).

\section{Figure 2: $\quad$ Stability study of $\Delta^{1}$-P6C/6-OH-PIP and 6-0xo-PIP}

Legend: A 4-month stability study was performed in urine samples from two subjects with pyridoxine dependent epilepsy. $\Delta^{1}$-piperideine-6-carboxyalte/6-hydroxy-pipecolate ( $\Delta^{1}-\mathrm{P} 6 \mathrm{C} / 6$ $\mathrm{OH}-\mathrm{PIP}$ ) and 6-oxo-pipecolate (6-oxo-PIP) concentrations were measured in three conditions: room temperature (circle), $-15^{\circ} \mathrm{C}$ (diamond) and $-70^{\circ} \mathrm{C}$ (square). Each sample was measured three times and reported as a mean value, and the mean was then represented as the percent of the initial concentration. At room temperature, $\Delta^{1}-\mathrm{P} 6 \mathrm{C} / 6-\mathrm{OH}-\mathrm{PIP}$ degraded quickly $(\mathrm{A})$ whereas 6-oxo-PIP was relatively stable for 126 days (B).

\section{Figure 3: $\quad$ Proposed modification to the lysine oxidation pathway}

Legend: In the revised lysine oxidation pathway both the pipecolate and the saccharopine pathway converge on the equilibrium of $\Delta^{1}$-piperideine-6-carboxylate ( $\left.\Delta^{1}-\mathrm{P} 6 \mathrm{C}\right)$ and 6-hydroxypipecolate (6-OH-PIP). The $\alpha$-aminoadipic semialdehyde ( $\alpha$-AASA) dehydrogenase enzyme acts on this metabolite to generate $\alpha$-AASA and then 2-amino-adipic acid (AAA). In $\alpha$-AASA dehydrogenase deficiency, a cytosolic enzyme catalyzed the oxidation of 6-OH-PIP to 6-oxopipecolate (6-oxo-PIP).

Abbreviations: $\mathrm{P} 2 \mathrm{C}, \Delta^{1}$-piperideine-2-carboxylate; $\mathrm{P} 2 \mathrm{H} 2 \mathrm{C}$, piperidine-2-hydroxy-2-carboxylate; $\mathrm{P} 2 \mathrm{CR}, \Delta^{1}$-piperideine-2-carboxylate reductase; PIP, pipecolic acid 


\section{References}

Al Teneiji A, Bruun TUJ, Cordeiro D, et al (2017) Phenotype, biochemical features, genotype and treatment outcome of pyridoxine-dependent epilepsy. Metab Brain Dis 32:443-451. doi: 10.1007/s11011-016-9933-8

Basura GJ, Hagland SP, Wiltse AM, Gospe SM (2009) Clinical features and the management of pyridoxine-dependent and pyridoxine-responsive seizures: review of 63 North American cases submitted to a patient registry. Eur J Pediatr 168:697-704. doi: 10.1007/s00431008-0823-x

Bok LA, Halbertsma FJ, Houterman S, et al (2012) Long-term outcome in pyridoxine-dependent epilepsy. Dev Med Child Neurol 54:849-854. doi: 10.1111/j.1469-8749.2012.04347.x

Brundidge SP, Gaeta FC, Hook DJ, et al (1980) Association of 6-oxo-piperidine-2-carboxylic acid with penicillin $\mathrm{V}$. Production on Penicillium chrysogenum fermentations. J Antibiot 33:1348-1351

Chan C-L, Wong JWY, Wong C-P, et al (2011) Human antiquitin: structural and functional studies. Chem Biol Interact 191:165-170. doi: 10.1016/j.cbi.2010.12.019

Coughlin CR, Swanson MA, Spector E, et al (2018) The genotypic spectrum of ALDH7A1 mutations resulting in pyridoxine dependent epilepsy: a common epileptic encephalopathy. J Inherit Metab Dis. doi: 10.1007/s10545-018-0219-7

Coughlin CR, van Karnebeek CDM, Al-Hertani W, et al (2015) Triple therapy with pyridoxine, arginine supplementation and dietary lysine restriction in pyridoxine-dependent epilepsy: Neurodevelopmental outcome. Mol Genet Metab. doi: 10.1016/j.ymgme.2015.05.011

Coulter-Mackie MB, Li A, Lian Q, et al (2012) Overexpression of human antiquitin in E. coli: enzymatic characterization of twelve ALDH7A1 missense mutations associated with pyridoxine-dependent epilepsy. Mol Genet Metab 106:478-481. doi: 10.1016/j.ymgme.2012.06.008

Coulter-Mackie MB, Tiebout S, van Karnebeek C, Stockler S (2014) Overexpression of recombinant human antiquitin in E. coli: partial enzyme activity in selected ALDH7A1 missense mutations associated with pyridoxine-dependent epilepsy. Mol Genet Metab 111:462-466. doi: 10.1016/j.ymgme.2014.02.010

Falsaperla R, Vari MS, Toldo I, et al (2018) Pyridoxine-dependent epilepsies: an observational study on clinical, diagnostic, therapeutic and prognostic features in a pediatric cohort. Metab Brain Dis 33:261-269. doi: 10.1007/s11011-017-0150-x

Farrant RD, Walker V, Mills GA, et al (2001) Pyridoxal phosphate de-activation by pyrroline-5carboxylic acid. Increased risk of vitamin B6 deficiency and seizures in hyperprolinemia type II. J Biol Chem 276:15107-15116. doi: 10.1074/jbc.M010860200

Garweg G, von Rehren D, Hintze U (1980) L-Pipecolate formation in the mammalian brain. Regional distribution of delta1-pyrroline-2-carboxylate reductase activity. J Neurochem 35:616-621 
Hallen A, Jamie JF, Cooper AJL (2013) Lysine metabolism in mammalian brain: an update on the importance of recent discoveries. Amino Acids 45:1249-1272. doi: 10.1007/s00726013-1590-1

Henriksen CM, Nielsen J, Villadsen J (1998) Cyclization of alpha-aminoadipic acid into the the delta-lactam 6-oxo-piperidine-2-carboxylic acid by Penicillium chrysogenum. J Antibiot 51:99-106

Hunt AD, Stokes J, McCRORY WW, Stroud HH (1954) Pyridoxine dependency: report of a case of intractable convulsions in an infant controlled by pyridoxine. Pediatrics 13:140-145

IJlst L, de Kromme I, Oostheim W, Wanders RJ (2000) Molecular cloning and expression of human L-pipecolate oxidase. Biochem Biophys Res Commun 270:1101-1105. doi: 10.1006/bbrc. 2000.2575

Jung S, Tran N-TB, Gospe SM, Hahn SH (2013) Preliminary investigation of the use of newborn dried blood spots for screening pyridoxine-dependent epilepsy by LC-MS/MS. Mol Genet Metab 110:237-240. doi: 10.1016/j.ymgme.2013.07.017

Mahajnah M, Corderio D, Austin V, et al (2016) A Prospective Case Study of the Safety and Efficacy of Lysine-Restricted Diet and Arginine Supplementation Therapy in a Patient With Pyridoxine-Dependent Epilepsy Caused by Mutations in ALDH7A1. Pediatr Neurol. doi: 10.1016/j.pediatrneurol.2016.03.008

Mathew EM, Moorkoth S, Lewis L, Rao P (2018) Biomarker Profiling for Pyridoxine Dependent Epilepsy in Dried Blood Spots by HILIC-ESI-MS. Int J Anal Chem 2018:2583215. doi: $10.1155 / 2018 / 2583215$

Meng Z, Lou Z, Liu Z, et al (2006) Crystal structure of human pyrroline-5-carboxylate reductase. J Mol Biol 359:1364-1377. doi: 10.1016/j.jmb.2006.04.053

Mercimek-Mahmutoglu S, Cordeiro D, Cruz V, et al (2014) Novel therapy for pyridoxine dependent epilepsy due to ALDH7A1 genetic defect: I-arginine supplementation alternative to lysine-restricted diet. Eur J Paediatr Neurol. doi: 10.1016/j.ejpn.2014.07.001

Mills PB, Struys E, Jakobs C, et al (2006) Mutations in antiquitin in individuals with pyridoxinedependent seizures. Nat Med 12:307-309. doi: 10.1038/nm1366

Nagy K, Takáts Z, Pollreisz F, et al (2003) Direct tandem mass spectrometric analysis of amino acids in dried blood spots without chemical derivatization for neonatal screening. Rapid Commun Mass Spectrom 17:983-990. doi: 10.1002/rcm.1000

Pena IA, Marques LA, Laranjeira ABA, et al (2016) Simultaneous detection of lysine metabolites by a single LC-MS/MS method: monitoring lysine degradation in mouse plasma. Springerplus 5:172. doi: 10.1186/s40064-016-1809-1

Pena IA, Marques LA, Laranjeira ÂBA, et al (2017) Mouse lysine catabolism to aminoadipate occurs primarily through the saccharopine pathway; implications for pyridoxine dependent epilepsy (PDE). Biochim Biophys Acta 1863:121-128. doi: 10.1016/j.bbadis.2016.09.006 
Porta F, Pagliardini V, Celestino I, et al (2017) Neonatal screening for biotinidase deficiency: A 30-year single center experience. Mol Genet Metab Rep 13:80-82. doi:

10.1016/j.ymgmr.2017.08.005

Posset R, Opp S, Struys EA, et al (2015) Understanding cerebral L-lysine metabolism: the role of L-pipecolate metabolism in Gcdh-deficient mice as a model for glutaric aciduria type I. J Inherit Metab Dis 38:265-272. doi: 10.1007/s10545-014-9762-z

Rothstein M, Miller LL (1954) The conversion of lysine to pipecolic acid in the rat. J Biol Chem 211:851-858

Ruekberg B, Rossoni E (2005) An Improved Preparation of 2,4-Dinitrophenylhydrazine Reagent. J Chem Educ 82:1310. doi: 10.1021/ed082p1310.2

Sadilkova K, Gospe SM, Hahn SH (2009) Simultaneous determination of alpha-aminoadipic semialdehyde, piperideine-6-carboxylate and pipecolic acid by LC-MS/MS for pyridoxine-dependent seizures and folinic acid-responsive seizures. J Neurosci Methods 184:136-141. doi: 10.1016/j.jneumeth.2009.07.019

Sauer SW, Opp S, Hoffmann GF, et al (2011) Therapeutic modulation of cerebral L-lysine metabolism in a mouse model for glutaric aciduria type I. Brain 134:157-170. doi: 10.1093/brain/awq269

Struys EA, Bok LA, Emal D, et al (2012) The measurement of urinary $\Delta^{1}$-piperideine-6carboxylate, the alter ego of $\alpha$-aminoadipic semialdehyde, in Antiquitin deficiency. $\mathrm{J}$ Inherit Metab Dis 35:909-916. doi: 10.1007/s10545-011-9443-0

Struys EA, Jakobs C (2007) a-Aminoadipic semialdehyde is the biomarker for pyridoxine dependent epilepsy caused by a-aminoadipic semialdehyde dehydrogenase deficiency. Molecular Genetics and Metabolism 91:405. doi: 10.1016/j.ymgme.2007.04.016

Struys EA, Jakobs C (2010) Metabolism of lysine in alpha-aminoadipic semialdehyde dehydrogenase-deficient fibroblasts: evidence for an alternative pathway of pipecolic acid formation. FEBS Lett 584:181-186. doi: 10.1016/j.febslet.2009.11.055

Struys EA, Jansen EEW, Salomons GS (2014) Human pyrroline-5-carboxylate reductase (PYCR1) acts on $\Delta(1)$-piperideine-6-carboxylate generating L-pipecolic acid. J Inherit Metab Dis 37:327-332. doi: 10.1007/s10545-013-9673-4

Suzuki M, West C, Beutler E (2001) Large-scale molecular screening for galactosemia alleles in a pan-ethnic population. Hum Genet 109:210-215

van Karnebeek CDM, Hartmann H, Jaggumantri S, et al (2012) Lysine restricted diet for pyridoxine-dependent epilepsy: first evidence and future trials. Mol Genet Metab 107:335-344. doi: 10.1016/j.ymgme.2012.09.006

Vashishtha AK, West AH, Cook PF (2015) Probing the chemical mechanism of saccharopine reductase from Saccharomyces cerevisiae using site-directed mutagenesis. Arch Biochem Biophys 584:98-106. doi: 10.1016/j.abb.2015.08.023 
Yuzyuk T, Liu A, Thomas A, et al (2016a) A novel method for simultaneous quantification of alpha-aminoadipic semialdehyde/piperideine-6-carboxylate and pipecolic acid in plasma and urine. J Chromatogr B Analyt Technol Biomed Life Sci 1017-1018:145-152. doi: 10.1016/j.jchromb.2016.02.043

Yuzyuk T, Thomas A, Viau K, et al (2016b) Effect of dietary lysine restriction and arginine supplementation in two patients with pyridoxine-dependent epilepsy. Mol Genet Metab 118:167-172. doi: 10.1016/j.ymgme.2016.04.015

Zheng XX, Shoffner JM, Voljavec AS, Wallace DC (1990) Evaluation of procedures for assaying oxidative phosphorylation enzyme activities in mitochondrial myopathy muscle biopsies. Biochim Biophys Acta 1019:1-10 